\title{
Experience in managing two challenging cases from paediatric age group
}

\section{Kallyan K.D ${ }^{1,2, *}$}

\section{${ }^{1}$ AIMST University, Kedah, Malaysia, ${ }^{2}$ (Past Affiliation) Hospital Duchess of Kent, Sabah, Malaysia}

For Correspondence *Kallyan Kishore Debnath, Email:kakideb@gmail.com Date of

Submisssion: 08-11-2019

Acceptance: 10-02-2019
Access this article online

Quick Response Code

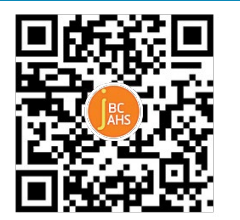

https://www.jbcahs.org

E-ISSN: 2581-6039

\section{ABSTRACT}

Mid-facial infection carries potential dangers of developing severe life-threatening intracranial complications. Early intervention and the awareness of possible complications essentially can help bring about a better outcome. Presented here, two paediatric cases of nasal abscesses, who developed such serious intracranial complications as meningitis, cavernous sinus thrombosis and brain infarction, but ultimately survived the crisis with appropriate intervening measures comprising immediate surgical intervention, efficient intensive care and long-term antibiotics. The cases were studied to ascertain a clinical course and outcome in the light of experience at a district hospital for further clinical application. No reconstructive surgery was performed on these patients but in short-term follow-up, no obvious facial developmental problem has been detected yet.

\section{Keywords:}

Mid-facial, nasal abscess, cavernous sinus, paediatric, danger triangle, intracranial, complication.

\section{INTRODUCTION}

Mid-facial or central face region is often referred to as the "danger triangle" of the face because of some special vascular characteristics of this area. ${ }^{1}$ Some veins of some individuals might not have valves. There is existence of communications between the facial vein and the cavernous sinus. The direction of blood flow also may favour the spread of infection from face to deeper intracranial structures. ${ }^{2}$ The infection in this area is not uncommon, but usually remain in a milder form. Severe form of infection, albeit rare, may lead to a retrograde spread intracranially. Cavernous sinus thrombosis (CST) is the late stage of such a complicating scenario posing a high mortality rate.

Nasal abscess, a kind of such central face infection, is not quite uncommon. Prevalence in male children is more than their female counterpart since they are more exposed to outdoor activities and thus vulnerable to more injuries.
Trauma (accidents, falls, nose picking, fights) is considered as the most common etiological factor for nasal abscess. ${ }^{3}$ Infection in and around the nose, such as nasal vestibulitis, furuncle, dental or sinus infection may also lead to abscess formation. Sometimes, abscess may develop spontaneously without any obvious detectable cause. ${ }^{4}$

Reasonably, the condition is more challenging to handle in the paediatric age-group, especially when the health care facilities are sub-optimal and/ or children's immunological status is compromised due to other associated underlying cause. However, knowledgeable primary health care professionals may play important role in managing such a case with their early and appropriate intervention.

\section{CASE N0. 1}

An eleven year old boy, a rural inhabitant and a known case of 
Figure 1: Case 1: A: at presentation, B: CT image brain (red arrows showing bilateral CST), C: at discharge, D: at follow up
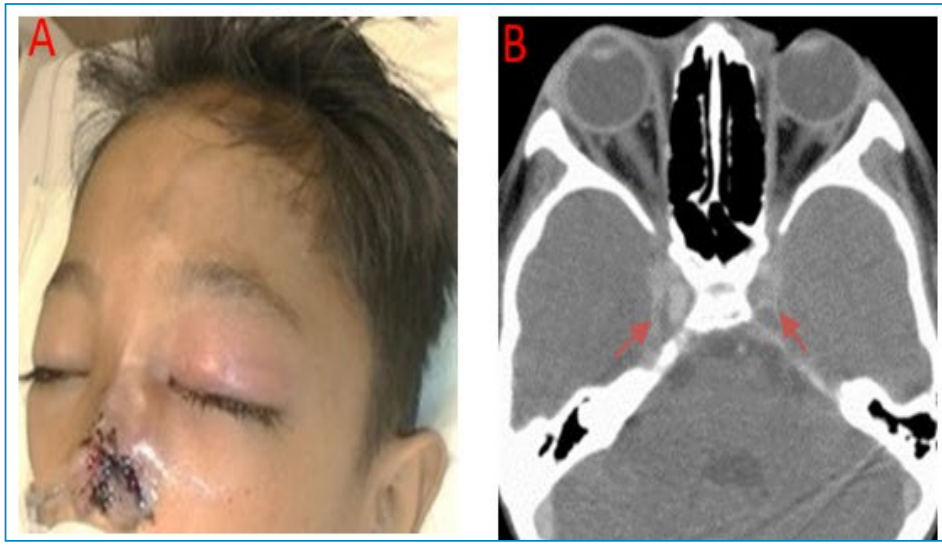
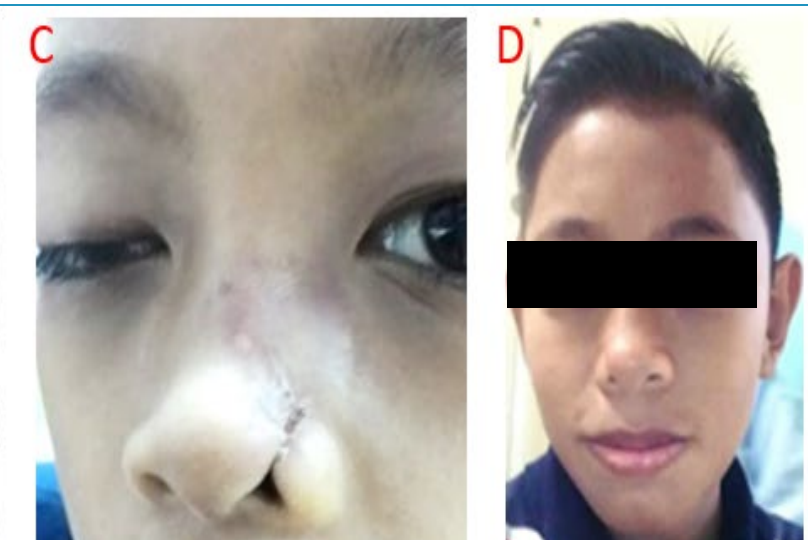

beta-thalassaemia major, presented with a painful nasal lesion for past 4 days and bilateral periorbital swellings associated with fever for 2 days (Figure 1). He had already been afflicted with anaemia and poor nutrition (body weight: $25 \mathrm{~kg}$ ). The problem began with a painful tiny lesion at the nasal tip which was then traumatised by one family member. The resultant cellulitis spread upwards assuming a well-formed abscess. Significant tissue necrosis brought up some nasal deformity. Both eye-lids were markedly oedematous and inflamed. Both pupils were moderately dilated with sluggish reaction to light. Rapid deterioration of Glasgow Coma Score (GCS) compelled the situation for ventilator and ionotropic support. Antibiotics (Table 1) were commenced empirically, coupled with concurrent surgical drainage of nasal abscess. Soon, patient developed weakness of left upper limb, bilateral keratitis, proptosis, ophthalmoplegia and ptosis (Rt.). Later, antibiotic was changed as per culture-sensitivity report of the collected specimens which showed growth
Table 1: Antibiotic therapies given to the patients

\begin{tabular}{|l|l|}
\hline \multicolumn{1}{|c|}{ Case No 1 } & \multicolumn{1}{c|}{ Case No 2} \\
\hline $\begin{array}{l}\text { IV Cloxacillin-2days } \\
\text { (until culture result is available) }\end{array}$ & $\begin{array}{l}\text { IV Ceftriaxone-3 } \\
\text { weeks } \\
\text { IV Ceftriaxone-10 days }\end{array}$ \\
IV Cloxacillin-3 weeks \\
IV Metronidazole- 5 days & $\begin{array}{l}\text { IV Metronidazole- } 5 \\
\text { days }\end{array}$ \\
IV Linezolid-21 days followed by & \\
oral Linezolid- 7 days & \\
\hline
\end{tabular}

of MRSA (Methicillin-resistant Staphylococcus aureus) for both blood and wound-swab.

The first CT-image was unremarkable whereas the second CT after one week showed bilateral CST with pre-septal cellulitis of left eye. The patient was discharged home safely after one month of treatment

Figure 2: Case 2: A: at presentation, B: CT image of brain, C: at follow-up

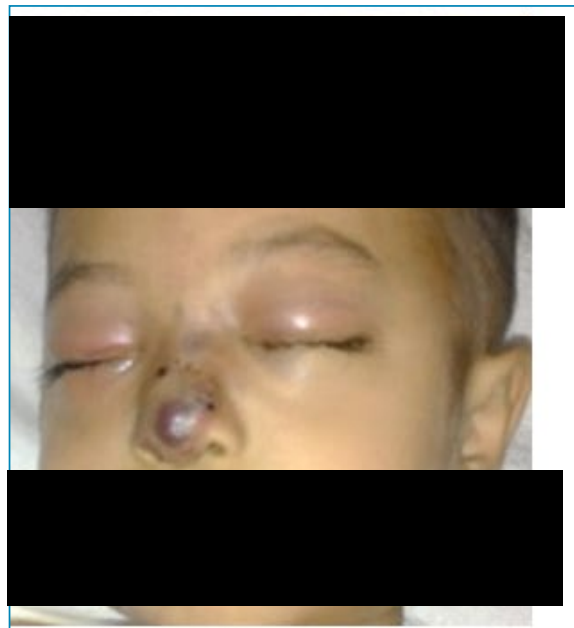

A

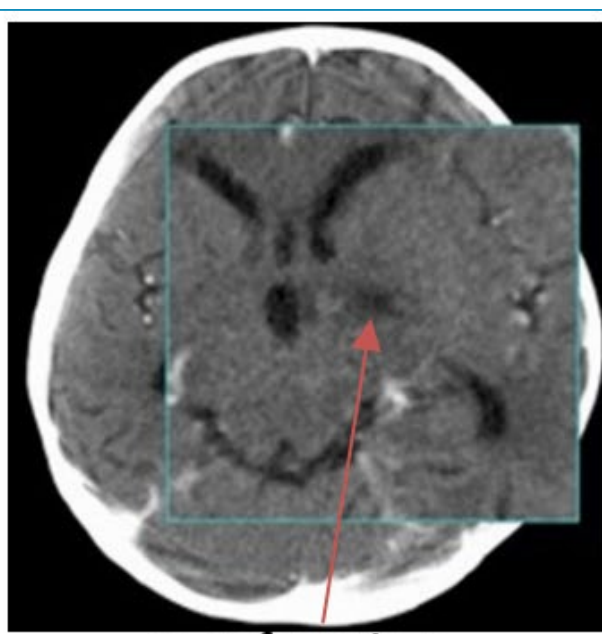

B: Infarction

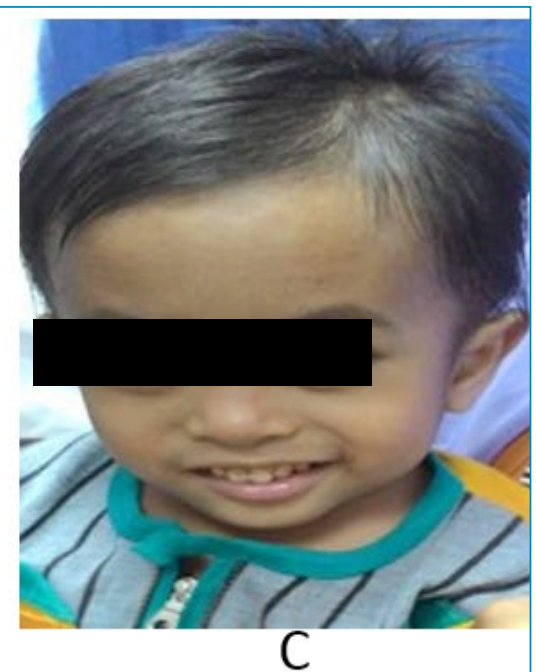


with minimal disabilities such as slight nasal deformity, right lateral rectus palsy and slight left upper limb weakness.

\section{CASE N0. 2}

Two year-old (body-weight: $8.5 \mathrm{~kg}$, Figure 2) child, hailing from rural area, presented with a painful lesion (with purulent discharge) at the nasal vestibule for past 3 days. It was associated with high grade fever. The other presenting features included difficult breathing, lethargy, drowsiness and irritability for past one day. The lesion was rapidly progressing over the dorsum of the nose up to both orbits causing marked periorbital oedema and swelling, later, leading to proptosis and ophthalmoplegia. The pupils were unequal and reacting abnormally, but Relative Afferent Pupillary Defect (RAPD) was negative bilaterally. The infective process spread both inward, to make a septal abscess and outward, to become evident as a subcutaneous spreading cellulitis causing the skin cracked, thinned out (bullous like) and bluish in colour. The condition was complicated further by moderate dehydration, hypokalaemia and metabolic acidosis.

Treatment was commenced immediately with same triple antibiotics (Table 1), coupled with immediate drainage procedure. The cultured bacteria (both blood and lesional pus showed growth of Staph. aureus) were sensitive to initially commenced antibiotics. But, even after one week of treatment, condition was not satisfactory. He developed ptosis, right sided motor weakness, facial palsy, keratitis with poor GCS $(<10)$. The first CT scan showed facial cellulitis and pansinusitis but no intracranial changes. It was only the second CT that exhibited the features of meningitis, infarction (left internal capsule) and right orbital abscess (extraconal collection with possible intraconal extension), but no CST. Another meticulous surgical procedure was required for this patient. Patient was discharged in 3 weeks' time with slight residual weakness in his right leg.

\section{DISCUSSION}

In both cases, the history of present illness was only a few days, that is alarming. Usually people do not seek treatment so early in this kind of common condition unless it is complicated. The pre-existing demographic status of both was vulnerable (rural children, anaemia, lack of family support/care, context of inadequate health awareness/personal hygiene etcetera). Initial trauma, considered quite common here, could be an exciting factor towards cellulitis. It seems reasonable that such a lesion better not to have disturbed without prior antibiotic coverage or close supervision. Though the lesions initially spread like cellulitis, the features of bullous appearance, skin break-down, rapid progression into deeper planes, significant tissue necrosis (involving cartilage) and hypotension might be consistent with necrotizing fasciitis. ${ }^{5}$ It's not sure, whether initial MRI could be more informative at early stage, although it would not change much our line of management. The sensitivity of contrast-CT and contrast-MRI could be comparable. ${ }^{6}$ However, repeated CTs were necessary. Since one of the patients got community-acquired MRSA, local bacteriological status (regarding antibiotic resistance) of a region should be considered while choosing an appropriate empirical antibiotic combination. High level of clinical suspicion and early recognition of increased intracranial pressure (e.g. abnormal pupillary size and/ or reaction) or other complications have important role. We did not use any anticoagulant; its beneficial effect is still questionable.

\section{CONCLUSION}

An awareness of serious complications is necessary among healthcare professionals, especially while dealing with vulnerable groups. For growing children, facial deformity and delayed facial growth could be additional concerns. ${ }^{4}$ In short to mid-term followup (3 - 4 years), no remarkable deformity has been documented in our cases; patient's family satisfaction is also quite good. However, further long-term follow up is required to ascertain the issue of developmental problem. High index of clinical suspicion, early institution of empirical antibiotics and good supportive care are the mainstays of treatment. Staph. aureus is commonly responsible, but possible resistance against common anti-staph antibiotics should be kept in mind. Waiting for a confirmatory diagnosis by investigations shouldn't make any undue delay in commencing utmost supportive care. Hence, a clinical astuteness is of prime importance.

\section{CONFLICTS OF INTEREST}

None 


\section{References}

1. Varshney S, Malhotra M, Gupta P, Gairola P, Kaur N. Cavernous sinus thrombosis of nasal origin in children. Indian J Otolaryngol Head Neck Surg. 2015;67:100-5.

2. Zhang J, Stringer MD. Ophthalmic and facial veins are not valveless. Clin Exp Ophthal. 2010;38:502-10.

3. Canty, PA, Berkowitz, RG. Hematoma and abscess of the nasal septum in children. Arch Otolaryngol Head Neck Surg. 1996; 122:1373-6.
4. Alshaikh N, Lo S. Nasal septal abscess in children: from diagnosis to management and prevention. Int J Pediatr Otorhinolaryngol. 2011;75:737-44.

5. Brook I. Microbiology and management of soft tissue and muscle infections. Int J Surg. 2008;6:328-38.

6. Smith DM, Vossough A, Vorona GA, Beslow LA, Ichord RN, Licht DJ. Pediatric cavernous sinus thrombosis: A case series and review of the literature. Neurology. 2015;85:763-9.

\section{Cleveland Clinic Unveils Top 10 Medical Innovations for 2019}

\section{Fighting the Opioid Crisis by Pharmacogenetic Testing}

Chronic pain is the leading cause of opioid prescription. Though several clinically validated alternative therapies for chronic pain exist, none have curtailed the crisis. Now, innovation and potential for hope comes by way of pharmacogenomic testing. In 2019, with increased access to genetic testing, pharmacogenomics is poised to make significant inroads into precision medicine and potentially an end to the crisis.

\section{The Advent of Artificial Intelligence (AI) in Healthcare}

AI is changing the game with its applications in decision support, image analysis and patient triage. It is helping physicians to make smarter decisions at the point of care, improving the ease and accuracy of viewing patient scans and reducing physician burnout.

\section{Expanded Window for Acute Stroke Intervention}

Until now, intervention of a stroke has only been recommended within a limited window of time. New guidelines suggest an expanded window for treatment. This lengthened timeframe is anticipated to lower the risk of disability and provide opportunity for recovery to an increased number of future stroke patients.

\section{Advances in Immunotherapy for Cancer Treatment}

Cancer immunotherapy, or biologic therapy, is a technique that uses the body's own immune system to fight cancer. With the near daily discovery of new immunotherapeutic targets and biomarkers, it is the hope that effective therapies will soon exist for all tumor profiles.

\section{Patient-Specific Products Achieved with 3D Printing}

The versatility provided by $3 \mathrm{D}$ printing gives medical practitioners the ability to provide patients the most advanced care, while minimizing the risk of complication. Currently, the most significant work includes external prosthetics, cranial/orthopedic implants, and customized airway stents for diseases narrowing the airway. To date, the technology has been used for many complicated heart surgeries, and even the most recent total face transplant.

\section{Virtual Reality and Mixed Reality (VR/MR) Technology for Medical Education}

VR/MR programs provide simulation training that serves to enhance traditional medical schooling. With this immersive style of learning, VR/MR training appeals to all types of learners: audio, visual and kinesthetic. Education via simulation is a productive step toward the system's most adept and confident healthcare providers.

\section{Visor for Prehospital Stroke Diagnosis}

Hemorrhagic strokes are responsible for nearly 40 percent of stroke deaths. Rapid diagnosis is necessary for effective treatment, as uncontrolled bleeding can lead to swelling of, and damage to, the brain. An efficient diagnostic tool, the visor for prehospital hemorrhage scanning serves to speed up diagnosis and the ever-important time to treatment.

\section{Innovation in Robotic Surgery}

Robots in the operating room provide surgeons with guidance for extreme precision in surgery. Shortened recovery time, limited pain after surgery and improved surgical outcomes are just a few of the patient benefits seen with robotized surgery.

\section{Mitral and Tricuspid Valve Percutaneous Replacement and Repair}

Cardiac surgery today is less invasive and more routine. Performed percutaneously - via a catheter through the skin many cardiac procedures no longer require an open heart surgery approach.

\section{RNA-Based Therapies}

Akin to DNA-based gene therapies, RNA-based therapies are the newest innovation in labs nationwide and have shown immense potential. Today, the most popular and successful mechanisms of RNA therapy include antisense nucleotides and RNA interference. These are being explored in a variety of rare genetic diseases such as Huntington's disease, as well as in cancer and neurologic diseases 